\title{
Pedicle subtraction osteotomy: a comprehensive analysis in 104 patients. Does the cause of deformity influence the outcome?
}

\author{
Karin Eskilsson, MD, ${ }^{1}$ Deep Sharma, MS, MCh, ${ }^{2}$ Christer Johansson, MSc, ${ }^{1}$ and \\ Rune Hedlund, $\mathrm{PhD}^{1}$
}

'Department of Orthopedics, Institution for Clinical Sciences, Sahlgrenska University Hospital, Gothenburg University, Gothenburg, Sweden; and 'Department of Orthopedics, Jawaharlal Institute of Postgraduate Medical Education and Research (JIPMER), Pondicherry, India

OBJECTIVE The clinical outcomes and complications of patients who underwent pedicle subtraction osteotomy (PSO) for various diagnoses were compared. More specifically, the purpose was to identify if outcomes differed between patients with flat-back syndrome after lumbar fusion (FBS-LF) versus patients who underwent surgery for adult spinal deformity (ASD).

METHODS A retrospective analysis of 104 patients who underwent a PSO for sagittal plane imbalance was performed. There were 28 patients with FBS-LF and 76 patients with various forms of ASD. Outcome was measured using visual analog scale (VAS)-back, VAS-leg, Oswestry Disability Index (ODI) (range 0-100 for all scales), and EQ-5D scores (range 0-1). Patients also rated their global outcomes as much better, better, unchanged, or worse at follow-up. The minimum follow-up was 1 year (range 1-4 years). Clinical outcomes and complications were compared between the 2 groups of patients.

RESULTS The most common level of PSO was L-3 and L-2; 100 single and 4 double PSOs were performed. The average local correction by PSO itself was $27.2^{\circ}$. The sagittal vertical axis (SVA) improved from a mean preoperative value of $74 \pm 23 \mathrm{~mm}$ to $49 \pm 20 \mathrm{~mm}$ at the final follow-up. The VAS-back, ODI, and EQ-5D scores improved significantly for the entire group by 33,16 , and 0.31 points, respectively. In total, $57 \%$ of patients reported that they were "much better" or "better" than before surgery. Preoperatively, as well as postoperatively, the FBS-LF patients reported significantly worse VAS scores. According to VAS-back results, the ASD group improved by 34 points compared with 29 points in FBS-LF patients. ODI scores were similar between the 2 groups preoperatively but improved significantly more in the ASD group (18 points) compared with the FBS-LF group (13 points). The EQ-5D scores improved from 0.07 to 0.35 in FBS-LF patients, and from 0.21 to 0.56 on average in ASD patients. Similarly, a "much better" or "better" outcome compared with before surgery was reported by $72 \%$ of patients in the ASD group compared with $24 \%$ of patients in the FBS-LF group ( $p$ $<0.001$ ). The overall reoperation rate was $31 \%$ : $46 \%$ of patients in the FBS-LF group compared with $25 \%$ of patients in the ASD group. There were $19(18 \%)$ dural tears, $14(13.5 \%)$ surgical site infections, $12(11.5 \%)$ instances of pseudarthrosis, $15(14 \%)$ proximal junctional failures, and 2 distal junctional failures. The $12(11 \%)$ neurological complications were dominated by partial weakness of hip flexion and knee extension, and all but 2 of these were temporary.

CONCLUSIONS PSO is a safe and effective method for correcting sagittal plane imbalance due to multiple etiologies. The authors found patient satisfaction to be high, and health-related quality of life was greatly improved by the procedure in patients with ASD. In contrast, in FBS-LF patients, a suboptimal outcome was observed and the cautious use of PSO seems warranted in this subset of patients.

https://thejns.org/doi/abs/10.3171/2016.12.SPINE16585

KEY WORDS pedicle subtraction osteotomy; adult spine deformity; vertebral osteotomy; lumbar flat back; kyphotic deformity

ABBREVIATIONS ASD = adult spinal deformity; FBS-LF = flat-back syndrome after lumbar fusion; ODI = Oswestry Disability Index; PJF = proximal junctional failure; PSO = pedicle subtraction osteotomy; SVA = sagittal vertical axis; VAS = visual analog scale.

SUBMITTED May 23, 2016. ACCEPTED December 15, 2016.

INCLUDE WHEN CITING Published online May 5, 2017; DOI: 10.3171/2016.12.SPINE16585. 
$\mathrm{S}$ agitTal plane spinal deformity has been increasingly recognized as an important cause of low health-related quality of life. It is commonly related to adult spinal deformity (ASD), i.e., degenerative scoliosis and adult idiopathic scoliosis, and also to various etiologies such as posttraumatic kyphosis and iatrogenic flat back after lumbar fusion. The clinical picture is characterized by back pain and often severe functional disability. Nonoperative treatment options fail in most patients. Surgical interventions, which are typically accomplished by extensive fusion with or without osteotomies, are aimed at restoring sagittal balance; perhaps the most common of these interventions is pedicle subtraction osteotomy (PSO). ${ }^{3,13}$

Originally, PSO was used in the ankylosed spine, particularly for ankylosing spondylitis, replacing open-up osteotomies or combined front and back surgery. ${ }^{13}$ Although it is a technically challenging method, satisfactory clinical outcomes have been reported. ${ }^{6,9,10}$ The indications for PSO currently include any kyphotic spinal disorder, such as degenerative scoliosis, posttraumatic kyphosis, neuromuscular scoliosis, congenital kyphoscoliosis, flat-back syndrome after previous scoliosis surgery, and flat-back syndrome after lumbar spine fusion (FBS-LF). ${ }^{9,14,15}$ The purpose of this study was to compare the clinical outcomes and complications in patients who underwent PSO for various spinal disorders and to identify possible differences in outcomes and complications between patients with a primary diagnosis of ASD compared with patients with FBS-LF for degenerative conditions.

\section{Methods}

A retrospective analysis of 104 consecutive patients who had undergone PSO between 2007 and 2012 at Sahlgrenska University Hospital in Gothenburg, Sweden, was performed. We obtained informed consent from all the patients and also approval from the ethics committee of Gothenburg University. The medical records were reviewed to extract demographic data (age, sex, height, and weight) and the preoperative diagnosis. The presence of any previous osteoporotic fracture and the history and nature of any previous spine surgery were also noted. Surgical data were extracted, including the level of PSO, proximal and distal level of instrumentation, operative time, estimated blood loss (including drainage), transfusions, and length of the hospital stay. The complications and their time of occurrence were recorded and classified as minor or major.

Before surgery, as part of our routine documentation, and at the final follow-up, the patients were asked to fill out validated questionnaires. Patients who did not send back the questionnaires were contacted by telephone and reminded to submit their questionnaires. Preoperative scores were available for all except 4 patients. The final follow-up scores were available for 90 (87\%) patients.

Pain was assessed using the visual analog scale (VAS) on a scale of 1-100 for back and leg pain separately, functional disability was assessed by the Oswestry Disability Index (ODI), and quality of life was assessed by EQ-5D. The global outcome ${ }^{8}$ as reported by the patients was classified as much better, better, unchanged, or worse at the final follow-up.

All patients underwent radiographic evaluation preoperatively, postoperatively, and at the final follow-up using Surgimap spine software. ${ }^{1}$ Full-length standing radiographs were obtained with documentation of the sagittal spinopelvic parameters, including sagittal vertical axis (SVA), thoracic kyphosis measured from T-4 to T-12, lumbar lordosis measured from L-1 to S-1, pelvic incidence, sacral slope, and pelvic tilt. All included patients were either in overt clinical sagittal imbalance (SVA $>5 \mathrm{~cm}$ ) or in compensating sagittal imbalance (pelvic tilt/sacral slope not corresponding to pelvic incidence, indicating pelvic retroversion or other types of compensatory changes ${ }^{12}$ ). The pedicle subtraction angle was defined as the change of the angle formed by the lower vertebral endplate of the adjacent cephalic vertebra to the upper vertebral endplate of the adjacent caudal vertebra.

The patients were grouped according to their diagnoses (Table 1). The 28 patients with FBS-LF were defined as the degenerative group (Fig. 1), and the 76 patients with a primary diagnosis of ASD were defined as the deformity group (Fig. 2). Among the patients with ASD, there were 20 patients with posttraumatic kyphosis, 16 patients

TABLE 1. Demographic data of 104 patients with multiple etiologies who underwent PSO

\begin{tabular}{lcccc}
\hline \multicolumn{1}{c}{ Diagnosis } & No. of Patients & Median Age at Op in Yrs (IQR) & \% Female & Median Body Mass Index (IQR) \\
\hline Degenerative group & & & & \\
\hline FBS-LF & 28 & $66(56-71)$ & 57 & $27(24-28)$ \\
\hline Deformity group & & & & \\
\hline Total & 76 & & & $24(22-29)$ \\
\hline Degenerative scoliosis & 21 & $63(58-70)$ & 76 & $26(23-29)$ \\
\hline Posttraumatic kyphosis & 20 & $63(56-68)$ & 70 & $25(24-28)$ \\
\hline Flat-back syndrome after scoliosis surgery & 16 & $49(25-56)$ & 100 & $26(23-30)$ \\
\hline Adolescent idiopathic scoliosis op as adult & 9 & $50(44-56)$ & 33 & $23(20-24)$ \\
\hline Neuromuscular scoliosis & 6 & $22(17-29)$ & 33 & $26(24-26)$ \\
\hline Congenital scoliosis & 3 & $24(18-46)$ & 0 & $22(12-33)$ \\
\hline Ankylosing spondylitis & 2 & $40(37-42)$ & 67 & $25(23-29)$ \\
\hline Total & 104 & $55(48-67)$ & & 75 \\
\hline
\end{tabular}


with flat-back syndrome following scoliosis surgery during adolescence using Harrington instrumentation, 21 patients with degenerative scoliosis, 9 patients with idiopathic scoliosis presenting in adulthood, 6 patients with neuromuscular scoliosis, 3 patients with congenital scoliosis, and 2 patients with ankylosing spondylitis. All patients had a kyphotic deformity assessed as severely affecting daily function.

\section{Statistical Analysis}

All statistical analyses were performed using SPSS (version 21, IBM SPSS, Inc.). Normally distributed variables were described using the mean \pm standard deviation, while variables not normally distributed were described using the median and interquartile range. The clinical outcomes and complication rates between the degenerative group and deformity group were analyzed for statistical significance using the t-test and chi-square tests. Statistical significance was set at $\mathrm{p}<0.05$.

\section{Results}

There were $33(32 \%)$ male and $71(68 \%)$ female patients, with a mean age of $55 \pm 16$ years. Seventy-five patients $(72 \%)$ had undergone previous spine surgery. The mean operative time was $336 \pm 89$ minutes (Table 2).

There were 100 single and 4 double PSOs. The most common PSO levels were L-3 (42\%) and L-2 (23\%). For PSOs at L-3, the most frequent instrumentation levels were T12-S1 (18\%), T4-S1 (16\%), and L1-5 (16\%). For PSOs at L-2, the most common instrumentation levels were T11-L5 (42\%) and T11-S1 (13\%). All patients had only 2 stabilizing rods across the PSO site. The mean (minimum-maximum) number of instrumented levels was 9 (5-17), mean intraoperative bleeding was $2600 \mathrm{ml}$ $(500 \mathrm{ml}$ to $10 \mathrm{~L}$ ), and mean postoperative drain collection was $900 \mathrm{ml}(100-2500 \mathrm{ml})$. Cell salvage with autologous transfusion was used in all patients. Perioperative blood transfusion was used in $72 \%$ (75 of 104) of patients, and postoperatively it was used in 32\% (33 of 104) of patients. The mean (minimum-maximum) length of hospitalization was 12 days (range 6-54). Patients with a flat-back diagnosis had on average a 7-level fusion compared with 10 levels in deformity patients.

All patients had a significant improvement in their clinical scores after PSO (Table 3). The average local correction by PSO itself was $27.2^{\circ}$, and the mean improvement in lumbar lordosis was from $29^{\circ}$ to $42^{\circ}$. SVA improved from a mean preoperative value of $74 \mathrm{~mm}$ to $49 \mathrm{~mm}$ at the follow-up ( $\mathrm{p}<0.001)$.

The mean preoperative and postoperative pain scores were significantly higher in the degenerative group (Table 4). The ODI scores were similar in the 2 groups preoperatively. However, after surgery, the improvement in ODI scores in the deformity group was significantly better (18\%) compared with the degenerative group (13\%). Similarly, the deformity group had significantly more improvement in the EQ-5D scores than the degenerative group (Table 4). On the global assessment scale, $72 \%$ of patients in the deformity group rated their status as "much better" or "better" (satisfactory outcome), whereas in the degen-
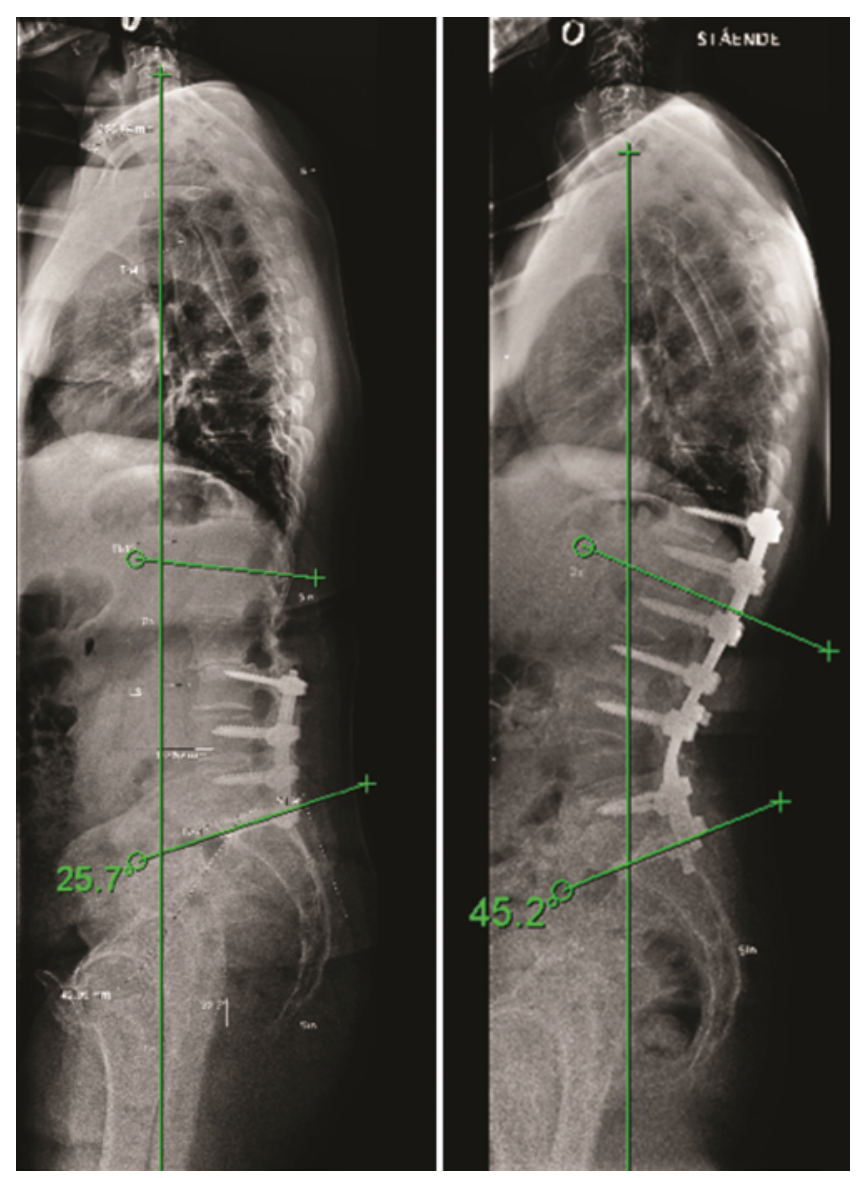

FIG. 1. Preoperative and postoperative radiographs of a 56 -year-old male patient who had previously undergone decompression and fusion at L3-S1. The patient developed flat-back syndrome and sagittal imbalance, an SVA of $8 \mathrm{~cm}$, and pelvic retroversion. He underwent reoperation 4 years later with PSO at L-4 and fusion at T11-S1. A normalized sagittal profile of the thoracic and lumbar spine as well as pelvic retroversion are shown. Figure is available in color online only.

erative group only $24 \%$ of patients reported a satisfactory outcome ( $\mathrm{p}<0.001)$ (Table 5).

Overall, there were $19(18 \%)$ intraoperative dural tears. Of $16(15.3 \%)$ surgical site infections, $10(9 \%)$ were deep and $6(5 \%)$ were superficial. The most common organism isolated was Staphylococcus aureus (8 patients), followed by Proteus mirabilis and Propionibacterium acnes in 2 patients each. There were $12(11.5 \%)$ instances of pseudarthrosis with rod breakage or implant failure. Pseudarthrosis occurred significantly more often in the degenerative group ( 8 of 28 patients; $28.5 \%$ ) than in the deformity group (4 of 76 patients; $5.3 \%$ ). If pseudarthrosis occurred after PSO, the revision included repairing pseudarthrosis by compression over the pseudarthrotic cleft, which often allowed some concomitant improvement in sagittal imbalance. Extension of fusion, typically superiorly, was performed if sagittal imbalance was not fully corrected. There were $15(14 \%)$ proximal junctional failures (PJFs) and $2(2 \%)$ distal junctional failures that required revision surgery in all of these patients, which involved extension of the instrumentation. PJF occurred 

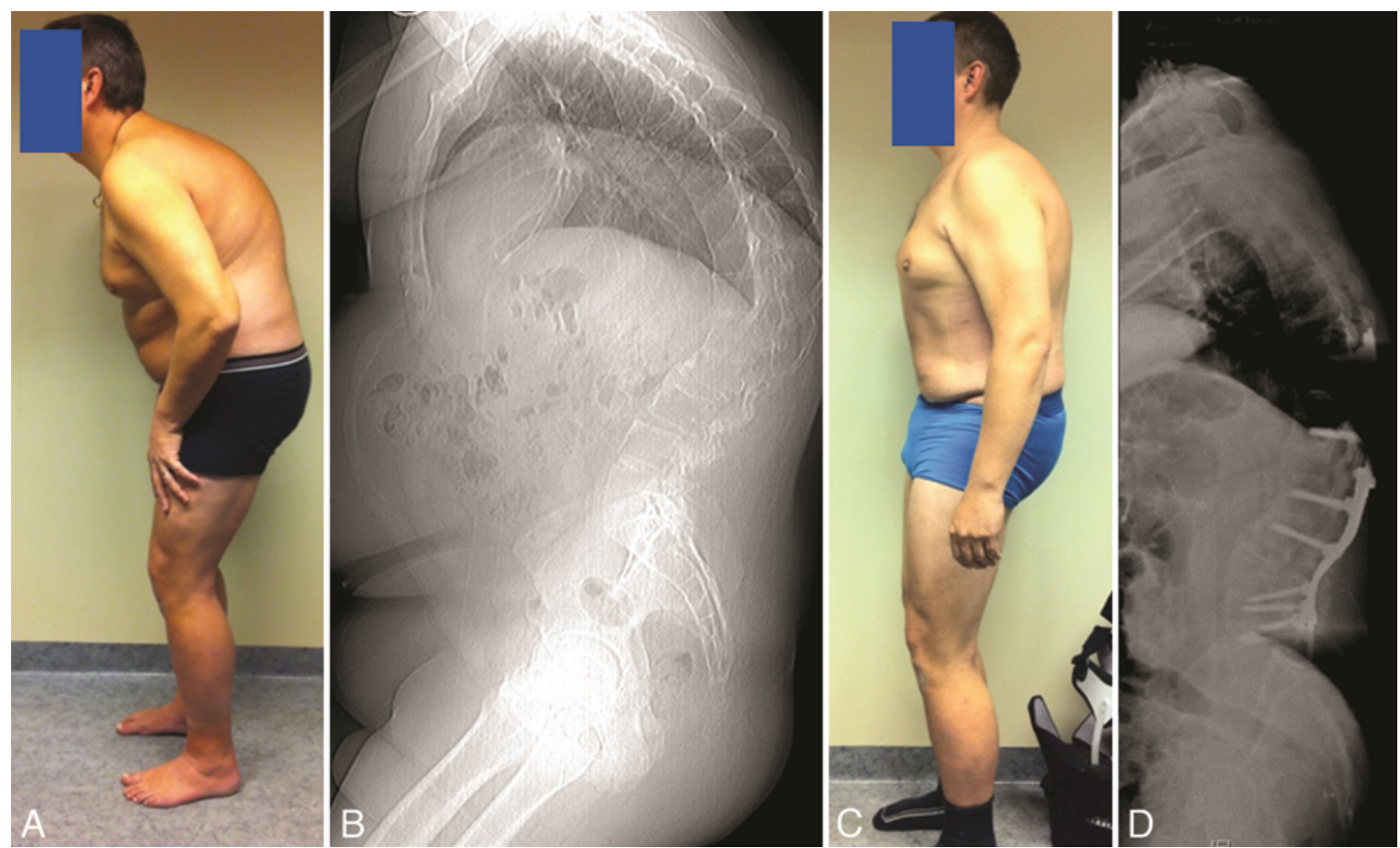

FIG. 2. Preoperative (A and $B$ ) and postoperative ( $C$ and $D)$ clinical photographs and radiographs obtained in a 42 -year-old male patient who was severely disabled and used a wheelchair outdoors due to ankylosing spondylitis and severe rigid kyphosis. He underwent double PSO at T-12 and L-3 and achieved an excellent clinical outcome. Figure is available in color online only.

significantly more frequently in the degenerative group (10 of 28 patients; $35.7 \%$ ) compared with the deformity group (5 of 76 patients; 6.5\%). Neurological complications occurred in $11 \%$ of the patients. The most common complication was weakness in hip flexion or knee extension. All but 2 patients recovered completely by the end of 1 year (Table 6).

The overall reoperation rate was $31 \%$ (32 of 104 patients). In the degenerative group, the reoperation rate was $46 \%$ (13 of 28 patients), which was significantly higher than in the deformity group (19 of 76 patients; $25 \%$ ). Reoperations in the deformity group were mostly performed on patients with adolescent idiopathic scoliosis who had undergone surgery as a child. These patients did as well or better than adults who underwent primary surgeries, and better than patients who underwent previous lumbar fusion as adults.

\section{Discussion}

We observed a striking difference in clinical outcomes between patients with various deformity diagnoses compared with the outcomes of patients with FBS-LF for degenerative lumbar disorders. Thus, the findings showed that outcome, to a large extent, was affected by the primary diagnosis. The most important finding was the strikingly poor outcome in FBS-LF patients, with only $24 \%$ of these patients demonstrating a satisfactory outcome and a mean change of only 0.27 on the EQ-5D index at the final follow-up. In contrast, $72 \%$ of patients with

TABLE 2. Surgical details and length of stay for the patients with different etiologies

\begin{tabular}{lcccc}
\hline \multicolumn{1}{c}{ Diagnosis } & $\begin{array}{c}\text { Median No. of } \\
\text { Instrumented Levels (IQR) }\end{array}$ & $\begin{array}{c}\text { Mean Op } \\
\text { Time in Mins }\end{array}$ & $\begin{array}{c}\text { Median Blood } \\
\text { Loss in ml }\end{array}$ & $\begin{array}{c}\text { Median Length of } \\
\text { Stay in Days (IQR) }\end{array}$ \\
\hline FBS-LF & $7(6-8)$ & 320 & 3600 & $11(9-17)$ \\
\hline Degenerative scoliosis & $8(7-9)$ & 322 & 2700 & $10(8-11)$ \\
\hline Posttraumatic kyphosis & $6(5-7)$ & 245 & 2300 & $9(8-12)$ \\
\hline FBS after scoliosis surgery & $15(8-15)$ & 377 & 3200 & $9(8-11)$ \\
\hline Adolescent idiopathic scoliosis op as adult & $13(8-14)$ & 391 & 3300 & $14(12-14)$ \\
\hline Neuromuscular scoliosis & $12(7-18)$ & 339 & 3000 & $12(10-14)$ \\
\hline Congenital scoliosis & $12(9-13)$ & 494 & 2400 & $11(10-17)$ \\
\hline Ankylosing spondylitis* & $11(9-13)$ & 391 & 8500 & $30(10-50)$ \\
\hline
\end{tabular}

\footnotetext{
* Includes only 2 patients.
} 
TABLE 3. Preoperative and postoperative outcome scores for all patients

\begin{tabular}{lcccc}
\hline Questionnaire & No. of Patients & Mean Preop & Mean Postop & p Value \\
\hline VAS-back & 90 & 70 & 37 & $0.005^{*}$ \\
\hline VAS-leg & 86 & 50 & 31 & $0.004^{*}$ \\
\hline ODI & 90 & 55 & 39 & $0.000^{*}$ \\
\hline EQ-5D index & 89 & 0.18 & 0.49 & $0.001^{*}$ \\
\hline
\end{tabular}

* The mean difference was significant at $p<0.05$

various classic spinal deformity diagnoses showed a satisfactory outcome and a mean change of 0.35 on EQ-5D. Also, compared with the ASD patients, the FBS-LF patients scored poorly on all of the clinical scores at the final follow-up, including VAS, ODI, EQ-5D, and the global assessment scale. Furthermore, the reoperation rate was almost double (46\%) in patients with previous lumbar fusion compared with the rate in patients with a primary deformity diagnosis (25\%). Thus, the findings show a much worse outcome in FBS-LF patients compared with ASD patients, who showed an overall satisfactory outcome in three-quarters of patients.

The reason for this outcome discrepancy is most likely multifactorial. Although they were all patients with sagittal imbalance, those undergoing PSO for the correction of FBS-LF differed considerably from the patients in whom the primary diagnosis was a spinal deformity. The difference is not only in the basic pathology, but also in the patient profiles between the 2 groups. FBS-LF patients, by definition, had previously undergone surgery for lowback pain, and it is likely that the selection of low-backpain patients whose surgeries had failed and who were in need of a reoperation results in a suboptimal outcome. A subgroup of patients in the deformity group-the patients who underwent surgery for adolescent idiopathic scoliosis as children also constitutes a reoperation group-did not undergo surgery initially for pain but for deformity. Thus,

TABLE 4. Difference in the clinical scores between the degenerative group and deformity group

\begin{tabular}{lccc}
\hline Outcome Variable & $\begin{array}{c}\text { Degenerative } \\
(\mathrm{n}=28)\end{array}$ & $\begin{array}{c}\text { Deformity } \\
(\mathrm{n}=76)\end{array}$ & $\mathrm{p}$ Value \\
\hline VAS-back & & & \\
\hline Preop & 77 & 68 & $0.021^{*}$ \\
\hline At last follow-up & 48 & 32 & $0.036^{*}$ \\
\hline Difference & 29 & 34 & 0.517 \\
\hline ODI & & & \\
\hline Preop & 59 & 54 & 0.211 \\
\hline At last follow-up & 46 & 36 & $0.002^{*}$ \\
\hline Difference & 13 & 18 & $0.039^{*}$ \\
\hline EQ-5D & & & \\
\hline Preop & 0.07 & 0.21 & $0.027^{*}$ \\
\hline At last follow-up & 0.35 & 0.56 & $0.004^{*}$ \\
\hline Difference & 0.28 & 0.35 & $0.024^{*}$ \\
\hline
\end{tabular}

* The mean difference was significant at $p<0.05$.
TABLE 5. Differences in global assessment between groups

\begin{tabular}{lcc}
\hline \multirow{2}{*}{ Global Outcome } & \multicolumn{2}{c}{ No. of Patients (\%) } \\
\cline { 2 - 3 } & Degenerative & Deformity \\
\hline Much better & $5(17)$ & $18(25)$ \\
\hline Better & $2(7)$ & $34(47)$ \\
\hline No change & $8(28)$ & $14(19)$ \\
\hline Worse & $14(48)$ & $7(9)$ \\
\hline Total no. of patients & 29 & 77 \\
\hline
\end{tabular}

they were not preselected pain patients, and most likely had a better chance of a satisfactory outcome.

Another reason for the major difference in outcome between the degenerative and deformity patients may be that the degenerative patients were predominantly fused up to the T10-L1 region, in contrast to the deformity patients who mostly underwent high thoracic fusion. The observed, much higher risk of PJF in the degenerative group (35\%) compared with PJF in the deformity group (7\%) strongly suggests that not only the diagnosis but also the length of fusion may affect the outcome. Furthermore, the

TABLE 6. Early and late major and minor complications

\begin{tabular}{|c|c|}
\hline Complications & No. of Patients \\
\hline \multicolumn{2}{|l|}{ Major complications } \\
\hline \multicolumn{2}{|l|}{ Intraop \& before discharge } \\
\hline Deep infection & 2 \\
\hline Motor deficit & 10 \\
\hline Pneumonia & 2 \\
\hline Pulmonary embolism & 1 \\
\hline Cervical fracture & 1 \\
\hline Iliac fracture & 1 \\
\hline Hematoma & 2 \\
\hline \multicolumn{2}{|l|}{ At final follow-up } \\
\hline Hematemesis & 1 \\
\hline Deep infection & 8 \\
\hline Implant failure & 15 \\
\hline Proximal junctional kyphosis & 15 \\
\hline Distal junctional kyphosis & 2 \\
\hline Sagittal imbalance & 3 \\
\hline Pseudarthrosis & 12 \\
\hline Total no. of major complications & 76 \\
\hline \multicolumn{2}{|l|}{ Minor complications } \\
\hline \multicolumn{2}{|l|}{ Intraop \& before discharge } \\
\hline Apathy & 1 \\
\hline CSF leak & 19 \\
\hline Superficial infection & 1 \\
\hline Excessive bleeding $>5 \mathrm{~L}$ & 20 \\
\hline Sensory deficit & 11 \\
\hline \multicolumn{2}{|l|}{ At final follow-up } \\
\hline Superficial infection & 5 \\
\hline Total no. of minor complications & 56 \\
\hline
\end{tabular}


degenerative patients were on average older (mean age 64 years) than the patients in the deformity group (mean age 52 years), with probably weaker musculature and poorer bone quality. This was reflected by the fact that $39 \%$ of the patients in the degenerative group had a history of osteoporotic vertebral fractures in contrast to only $18 \%$ of the patients in the deformity group. Also, the presence of other comorbidities in these older patients may have had an impact on their clinical outcomes.

Also, pseudarthrosis occurred more often in the patients with preoperative flat backs. Of the 12 total cases of pseudoarthrosis, 8 were seen in the degenerative group and 4 in the deformity group. Of these, 6 were at the level of the osteotomy. All patients with pseudoarthrosis underwent revision and fusion was accomplished. In the present study, PSO was a part of the learning curve for some surgeons, particularly in the degenerative group, which possibly influenced the results.

There are no previous comparisons between the outcomes of PSO in patients with FBS-LF and patients with more traditional ASD. Somewhat in contrast to our study, Kim et al., ${ }^{9}$ in their retrospective review of 140 patients who underwent PSO for various etiologies, reported no significant differences in the clinical outcomes or complications between various subgroups. However, 61\% (86 of 140) of their patients had ankylosing spondylitis, thereby rendering comparison difficult. Gupta et al., in a multicentric study comparing outcomes after PSO as a primary versus revision surgery in patients with ASD, demonstrated similar correction and complication rates in both groups, except for pseudarthrosis, which was seen more commonly in a revision setting. They, however, did not make a distinction between the various etiological diagnoses and also did not report patient-based, health-related quality of life measures. In the present study, we did not compare primary versus revision surgery in ASD patients due to the limited number of revisions for ASD.

Similar to the published literature, ${ }^{3,5,7,9,10}$ we noted a high rate of complications. Neurological complications were seen in $11 \%$ patients, which is comparable to that in previous studies. ${ }^{5}$ Most complications were mild and reversible. There were no deaths related to the surgical procedure. Intraoperative blood loss was highly variable, with excessive bleeding ( $>5000 \mathrm{ml}$ ) occurring in $19 \%$ of patients. The median bleeding of $2000 \mathrm{ml}$ is similar to other studies ${ }^{9}$ and can be considered large, but was tolerable for the great majority of patients with only 1 patient with a complication that could be attributed to blood loss. Other than pseudarthrosis and PJF, there were no significant differences in the complication rates between the 2 groups.

Largely in agreement with our study, most authors $\mathbf{s}^{7,9,10}$ have reported that PSO is a safe and effective method for correcting sagittal malalignment. The average observed local correction obtained by PSO $\left(27^{\circ}\right)$ is similar to a previously reported study. ${ }^{4}$ The mean preoperative lumbar lordosis was increased by only $13^{\circ}$. The much smaller increase in lumbar lordosis than the local correction obtained by PSO is explained by the fact that some of the PSOs were performed at the thoracic level, and if local midlumbar lordosis were corrected by PSO then the compensatory low lumbar lordosis actually was reduced in some patients because the need for compensatory changes below the PSO was no longer required. This resulted in a more harmonious sagittal profile. In contrast to previous studies that reported mainly radiographic results, the present study focused on clinical outcome and complications according to diagnosis. Although extensive radiographic analyses were performed with quantification of the spinopelvic variables, we decided not to include these data because of the risk of obscuring the major findings, i.e., the variation in clinical outcomes between different diagnoses. A detailed analysis of sagittal balance is presented separately.

The strength of our study is the large sample size and the extent of the data that were collected on the demographics, clinical evaluations, complications, and patientbased, health-related quality of life. The presence of consistent findings across multiple validated outcome tools increases the statistical confidence and clinical relevance of the findings. However, apart from the retrospective study design, there are several limitations. The relatively shorter follow-up period ranged from 1 to 4 years and may not be adequate for estimating outcomes and the complication rate. This is particularly true for pseudarthrosis; it is reported that $40 \%-50 \%$ of cases may present later than 2 years postoperatively. ${ }^{10,11}$ However, the maximum improvement in the health-related quality of life of a patient is reported to occur during the first 6 months following surgery, even in the presence of complications. ${ }^{2}$ Thus, a longer follow-up period may not yield significantly different clinical outcomes than those achieved at the end of 1 year. An additional limitation is the limited number of patients in some subgroups. The similar outcomes observed in the different types of deformity patients, however, justify the combined analysis of this group to some extent. A separate analysis of each diagnostic subgroup would have been preferable because they do differ in many important aspects. However, the limited number of patients in the different subgroups does not allow any conclusion from such an analysis. It may of course be that such differences exist, but a much larger series of patients is needed to study this possibility.

\section{Conclusions}

PSO is a safe and effective method for correcting sagittal plane imbalance due to multiple etiologies. We found patient satisfaction to be high, and health-related quality of life was greatly improved by the procedure in patients with ASD. In contrast, a suboptimal outcome was observed in patients with FBS-LF, and the cautious use of the PSO seems warranted in this subset of patients.

\section{References}

1. Akbar M, Terran J, Ames CP, Lafage V, Schwab F: Use of Surgimap Spine in sagittal plane analysis, osteotomy planning, and correction calculation. Neurosurg Clin N Am 24:163-172, 2013

2. Ayhan S, Aykac B, Yuksel S, Guler UO, Pellise F, Alanay A, et al: Safety and efficacy of osteotomies in adult spinal deformity: what happens in the first year? Eur Spine J 25:24712479, 2016

3. Bridwell KH, Lewis SJ, Edwards C, Lenke LG, Iffrig TM, 
Berra A, et al: Complications and outcomes of pedicle subtraction osteotomies for fixed sagittal imbalance. Spine (Phila Pa 1976) 28:2093-2101, 2003

4. Bridwell KH, Lewis SJ, Lenke LG, Baldus C, Blanke K: Pedicle subtraction osteotomy for the treatment of fixed sagittal imbalance. J Bone Joint Surg Am 85-A:454-463, 2003

5. Buchowski JM, Bridwell KH, Lenke LG, Kuhns CA, Lehman RA Jr, Kim YJ, et al: Neurologic complications of lumbar pedicle subtraction osteotomy: a 10-year assessment. Spine (Phila Pa 1976) 32:2245-2252, 2007

6. Cho KJ, Bridwell KH, Lenke LG, Berra A, Baldus C: Comparison of Smith-Petersen versus pedicle subtraction osteotomy for the correction of fixed sagittal imbalance. Spine (Phila Pa 1976) 30:2030-2038, 2005

7. Gupta MC, Ferrero E, Mundis G, Smith JS, Shaffrey CI, Schwab F, et al: Pedicle subtraction osteotomy in the revision versus primary adult spinal deformity patient: is there a difference in correction and complications? Spine (Phila Pa 1976) 40:E1169-E1175, 2015

8. Hägg O, Fritzell P, Odén A, Nordwall A: Simplifying outcome measurement: evaluation of instruments for measuring outcome after fusion surgery for chronic low back pain. Spine (Phila Pa 1976) 27:1213-1222, 2002

9. Kim KT, Lee SH, Suk KS, Lee JH, Jeong BO: Outcome of pedicle subtraction osteotomies for fixed sagittal imbalance of multiple etiologies: a retrospective review of 140 patients. Spine (Phila Pa 1976) 37:1667-1675, 2012

10. Kim YJ, Bridwell KH, Lenke LG, Cheh G, Baldus C: Results of lumbar pedicle subtraction osteotomies for fixed sagittal imbalance: a minimum 5-year follow-up study. Spine (Phila Pa 1976) 32:2189-2197, 2007

11. O'Neill KR, Lenke LG, Bridwell KH, Hyun SJ, Neuman B, Dorward I, et al: Clinical and radiographic outcomes after 3-column osteotomies with 5-year follow-up. Spine (Phila Pa 1976) 39:424-432, 2014

12. Schwab F, Lafage V, Patel A, Farcy JP: Sagittal plane consid- erations and the pelvis in the adult patient. Spine (Phila Pa 1976) 34:1828-1833, 2009

13. Thomasen E: Vertebral osteotomy for correction of kyphosis in ankylosing spondylitis. Clin Orthop Relat Res (194):142152,1985

14. Xi YM, Pan M, Wang ZJ, Zhang GQ, Shan R, Liu YJ, et al: Correction of post-traumatic thoracolumbar kyphosis using pedicle subtraction osteotomy. Eur J Orthop Surg Traumatol 23 (Suppl 1):S59-S66, 2013

15. Zeng Y, Chen Z, Sun C, Li W, Qi Q, Guo Z, et al: Posterior surgical correction of posttraumatic kyphosis of the thoracolumbar segment. J Spinal Disord Tech 26:37-41, 2013

\section{Disclosures}

Dr. Hedlund reports that he receives research funds from Zimmer and DePuy Synthes and is on the speakers' bureau for Zimmer, Globus, K2M, and DePuy Synthes.

\section{Author Contributions}

Conception and design: Hedlund. Acquisition of data: Eskilsson, Johansson. Analysis and interpretation of data: Sharma, Eskilsson, Hedlund. Drafting the article: Sharma, Eskilsson, Hedlund. Critically revising the article: Sharma, Hedlund. Reviewed submitted version of manuscript: Sharma. Approved the final version of the manuscript on behalf of all authors: Sharma. Statistical analysis: Sharma, Eskilsson, Johansson. Study supervision: Hedlund.

\section{Correspondence}

Deep Sharma, Department of Orthopedics, Jawaharlal Institute of Postgraduate Medical Education and Research (JIPMER), Pondicherry 605006, India. email: drdeep_sharma@yahoo.com. 